\title{
Front Matter: Volume 8984
}

, "Front Matter: Volume 8984," Proc. SPIE 8984, Ultrafast Phenomena and Nanophotonics XVIII, 898401 (19 March 2014); doi: 10.1117/12.2063509

SPIE. Event: SPIE OPTO, 2014, San Francisco, California, United States 


\title{
PROCEEDINGS OF SPIE
}

\section{Ultrafast Phenomena and Nanophotonics XVIII}

\author{
Markus Betz \\ Abdulhakem Y. Elezzabi \\ Jin-Joo Song \\ Kong-Thon Tsen \\ Editors
}

2-5 February 2014

San Francisco, California, United States

Sponsored by

SPIE

Cosponsored by

Femtolasers, Inc. (United States)

Published by

SPIE 
The papers included in this volume were part of the technical conference cited on the cover and title page. Papers were selected and subject to review by the editors and conference program committee. Some conference presentations may not be available for publication. The papers published in these proceedings reflect the work and thoughts of the authors and are published herein as submitted. The publisher is not responsible for the validity of the information or for any outcomes resulting from reliance thereon.

Please use the following format to cite material from this book:

Author(s), "Title of Paper," in Ultrafast Phenomena and Nanophotonics XVIII, edited by Markus Betz, Abdulhakem Y. Elezzabi, Jin-Joo Song, Kong-Thon Tsen, Proceedings of SPIE Vol. 8984 (SPIE, Bellingham, WA, 2014) Article CID Number.

ISSN: 0277-786X

ISBN: 9780819498977

Published by

SPIE

P.O. Box 10, Bellingham, Washington 98227-0010 USA

Telephone +1 3606763290 (Pacific Time) · Fax +1 3606471445

SPIE.org

Copyright (C) 2014, Society of Photo-Optical Instrumentation Engineers.

Copying of material in this book for internal or personal use, or for the internal or personal use of specific clients, beyond the fair use provisions granted by the U.S. Copyright Law is authorized by SPIE subject to payment of copying fees. The Transactional Reporting Service base fee for this volume is $\$ 18.00$ per article (or portion thereof), which should be paid directly to the Copyright Clearance Center (CCC), 222 Rosewood Drive, Danvers, MA 01923. Payment may also be made electronically through CCC Online at copyright.com. Other copying for republication, resale, advertising or promotion, or any form of systematic or multiple reproduction of any material in this book is prohibited except with permission in writing from the publisher. The CCC fee code is 0277-786X/14/\$18.00.

Printed in the United States of America.

Publication of record for individual papers is online in the SPIE Digital Library.

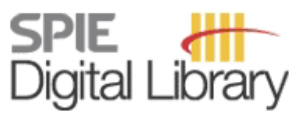

SPIEDigitalLibrary.org

Paper Numbering: Proceedings of SPIE follow an e-First publication model, with papers published first online and then in print and on CD-ROM. Papers are published as they are submitted and meet publication criteria. A unique, consistent, permanent citation identifier (CID) number is assigned to each article at the time of the first publication. Utilization of CIDs allows articles to be fully citable as soon as they are published online, and connects the same identifier to all online, print, and electronic versions of the publication. SPIE uses a six-digit CID article numbering system in which:

- The first four digits correspond to the SPIE volume number.

- The last two digits indicate publication order within the volume using a Base 36 numbering

system employing both numerals and letters. These two-number sets start with 00, 01, 02, 03, 04, $05,06,07,08,09,0 A, 0 B \ldots 0 Z$, followed by 10-1Z, 20-2Z, etc.

The CID Number appears on each page of the manuscript. The complete citation is used on the first page, and an abbreviated version on subsequent pages. Numbers in the index correspond to the last two digits of the six-digit CID Number. 


\section{Contents}

ix Conference Committee

\section{SESSION 1 NONLINEAR OPTICAL PHENOMENA}

898404 Hyper Rayleigh scattering from gold nanorods: The shape effect for centrosymmetric nanoparticles [8984-3]

I. Russier-Antoine, Institut Lumière Matière, CNRS, Univ. Claude Bernard Lyon 1 (France);

A. McLintock, A. W. Wark, Univ. of Strathclyde (United Kingdom); F. Bertorelle, E. Benichou,

E. Bergman, A. Bruyère, C. Jonin, P.-F. Brevet, Institut Lumière Matière, CNRS, Univ. Claude

Bernard Lyon 1 (France)

\section{SESSION 2 ACTIVE PLASMONICS}

898408 Ultrafast two-photon absorption generated free-carrier modulation in a silicon nanoplasmonic resonator [8984-7]

M. P. Nielsen, A. Y. Elezzabi, Univ. of Alberta (Canada)

898409 Active plasmonics: merging metals with semiconductors (Invited Paper) [8984-8] P. Vasa, Carl von Ossietzky Univ. Oldenburg (Germany) and Indian Institute of Technology Bombay (India); W. Wang, R. Pomraenke, Carl von Ossietzky Univ. Oldenburg (Germany); M. Maiuri, C. Manzoni, G. Cerullo, Istituto di Fotonica e Nanotecnologie, CNR, Politecnico di Milano (Italy); C. Lienau, Carl von Ossietzky Univ. Oldenburg (Germany)

\section{SESSION 3 THZ PHENOMENA}

8984 OB Feasibility of GaN-based room temperature THz laser in a spoof plasmon waveguide (Invited Paper) [8984-10]

G. Sun, Univ. of Massachusetts, Boston (United States); J. B. Khurgin, Johns Hopkins Univ. (United States); D. P. Tsai, National Taiwan Univ. (Taiwan)

8984 OC THz acoustic spectroscopy based on GaN nanostructures (Invited Paper) [8984-11] K.-H. Lin, D.-H. Tsai, K.-J. Wang, Academia Sinica (Taiwan); S.-H. Chen, K.-L. Chi, J.-W. Shi, National Central Univ. (Taiwan); P.-C. Chen, J.-K. Sheu, National Cheng Kung Univ. (Taiwan)

8984 OD Modeling of ultrafast THz interactions in molecular crystals [8984-12] P. Klarskov, Technical Univ. of Denmark (Denmark); S. J. Clark, Durham Univ. (United Kingdom); P. U. Jepsen, Technical Univ. of Denmark (Denmark)

8984 OF Optical response of tightly coupled THz metamaterials [8984-14] J.-H. Kang, Q.-H. Park, Korea Univ. (Korea, Republic of) 
8984 Ol Si-based nanoplasmonic resonant devices for all-optical integrated circuits [8984-17] M. P. Nielsen, A. Y. Elezzabi, Univ. of Alberta (Canada)

\section{SESSION 5 ULTRAFAST DYNAMICS IN CARBON NANOMATERIALS}

8984 OK Terahertz and ultrafast dynamics of carriers and phonons in graphene and carbon nanotubes (Invited Paper) [8984-19]

W. Gao, Q. Zhang, L. Ren, Z. Jin, Rice Univ. (United States); J.-H. Kim, Sungkyunkwan Univ. (Korea, Republic of); J. Kono, Rice Univ. (United States)

8984 OL Carrier multiplication and optical gain in graphene (Invited Paper) [8984-20]

T. Winzer, E. Malic, Technische Univ. Berlin (Germany)

\section{SESSION 6 COHERENT OPTICAL PHENOMENA I}

8984 ON Optical three-dimensional coherent spectroscopy (Invited Paper) [8984-22]

H. Li, JILA, Univ. of Colorado, NIST (United States) and Florida International Univ. (United States); A. D. Bristow, JILA, Univ. of Colorado, NIST (United States) and West Virginia Univ. (United States); M. E. Siemens, JILA, Univ. of Colorado, NIST (United States) and Univ. of Denver (United States); G. Moody, S. T. Cundiff, JILA, Univ. of Colorado, NIST (United States) and Univ. of Colorado (United States)

\section{SESSION 7 COHERENT OPTICAL PHENOMENA II}

8984 OS Spectral and temporal characteristics of a transient Cherenkov radiation from a periodic resonant medium excited by an ultrashort laser pulse at superluminal velocity [8984-27] R. Arkhipov, Weierstrass-Institut für Angewandte Analysis und Stochastik (Germany) and St. Petersburg State Univ. (Russian Federation); I. Babushkin, Humboldt-Univ. zu Berlin (Germany); M. V. Arkhipov, Y. A. Tolmachev, St. Petersburg State Univ. (Russian Federation)

\section{SESSION 8 NANOPHOTONICS}

8984 OU Ultrafast optical microscopy on single semiconductor nanowires (Invited Paper) [8984-29] M. Seo, Los Alamos National Lab. (United States) and Korea Institute of Science and Technology (Korea, Republic of); J. Yoo, S. A. Dayeh, S. T. Picraux, A. J. Taylor, R. P. Prasankumar, Los Alamos National Lab. (United States)

8984 OV Principles of perfect and ultrathin anti-reflection with applications to transparent electrode (Invited Paper) [8984-30]

K.-H. Kim, Q.-H. Park, Korea Univ. (Korea, Republic of) 
8984 OW Phase space monitoring of exciton-polariton multistability (Invited Paper) [8984-31]

Y. Léger, Foton Lab., CNRS-INSA, Univ. Europeenne de Bretagne (France)

8984 OX Formation and control of transverse patterns in a quantum fluid of microcavity polaritons [8984-32]

P. Lewandowski, Univ. Paderborn (Germany); V. Ardizzone, Lab. Pierre Aigrain, École Normale Supériere, CNRS, Univ. Pierre et Marie Curie, Univ. D. Diderot (France); Y. C. Tse, The Chinese Univ. of Hong Kong (China); N. H. Kwong, The Chinese Univ. of Hong Kong (China) and College of Optical Sciences, The Univ. of Arizona (United States); M. H. Luk, The Chinese Univ. of Hong Kong (China) and The Univ. of Arizona (United States); A. Lücke, Univ. Paderborn (Germany); M. Abbarchi, Lab. Pierre Aigrain, École Normale Supériere, CNRS, Univ. Pierre et Marie Curie, Univ. D. Diderot (France) and Lab. de Photonique et de Nanostructures, CNRS (France); J. Bloch, Lab. de Photonique et de Nanostructures, CNRS (France); E. Baudin, Lab. Pierre Aigrain, École Normale Supériere, CNRS, Univ. Pierre et Marie Curie, Univ. D. Diderot (France); E. Galopin, A. Lemaître, Lab. de Photonique et de Nanostructures, CNRS (France); P. T. Leung, The Chinese Univ. of Hong Kong (Hong Kong, China); Ph. Roussignol, Lab. Pierre Aigrain, École Normale Supériere, CNRS, Univ. Pierre e† Marie Curie, Univ. D. Diderot (France); R. Binder, College of Optical Sciences, The Univ. of Arizona (United States); J. Tignon, Lab. Pierre Aigrain, École Normale Supériere, CNRS, Univ. Pierre et Marie Curie, Univ. D. Diderot (France); S. Schumacher, Univ. Paderborn (Germany) and College of Optical Sciences, The Univ. of Arizona (United States)

\section{SESSION $10 \quad$ THZ DETECTION AND IMAGING}

898410 THz detection in graphene nanotransistors (Invited Paper) [8984-35]

A. Tredicucci, M. S. Vitiello, M. Polini, NEST, CNR-Istituto Nanoscienze and Scuola Normale Superiore (Italy); V. Pellegrini, NEST, CNR-Istituto Nanoscienze and Scuola Normale Superiore (Italy) and Istituto Italiano di Tecnologia (Italy)

898411 Imaging ultrafast dynamics on the nanoscale with a THz-STM (Invited Paper) [8984-36]

T. L. Cocker, V. Jelic, J. R. Hoffman, M. Gupta, R. Miller, S. J. Molesky, J. A. J. Burgess, G. De Los Reyes, L. V. Titova, Y. Y. Tsui, M. R. Freeman, F. A. Hegmann, Univ. of Alberta (Canada)

898412 Ultrafast THz-pulse-induced tunneling dynamics in an STM [8984-37]

V. Jelic, Univ. of Alberta (Canada); T. L. Cocker, Univ. of Alberta (Canada) and Univ. of Regensburg (Germany); J. R. Hoffman, M. Gupta, R. Miller, S. J. Molesky, J. A. J. Burgess, G. B. De Los Reyes, L. V. Titova, Y. Y. Tsui, M. R. Freeman, F. A. Hegmann, Univ. of Alberta (Canada)

898413 Broadband THz imaging in gas and multiple quantum-well media [8984-38] C.-Y. Li, The Univ. of New Mexico (United States); D. V. Seletskiy, Univ. Konstanz (Germany) and The Univ. of New Mexico (United States); J. G. Cederberg, Sandia National Labs. (United States); M. Sheik-Bahae, The Univ. of New Mexico (United States)

898414 Silicon wafer thickness measurement using terahertz time domain spectroscopy main [8984-39]

C.-Y. Jen, C. Richter, Rochester Institute of Technology (United States) 
898415 Ultrafast spin precession and transport controlled and probed with terahertz radiation (Invited Paper) [8984-40]

T. Kampfrath, Fritz-Haber-Institut der Max-Planck-Gesellschaft (Germany); M. Battiato, Uppsala Univ. (Sweden); A. Sell, Univ. Konstanz (Germany); F. Freimuth, Peter-GrunbergInstitut and Institute for Advanced Simulation (Germany); A. Leitenstorfer, Univ. Konstanz (Germany); M. Wolf, Fritz-Haber-Institut der Max-Planck-Gesellschaft (Germany); R. Huber, Univ. Regensburg (Germany); P. M. Oppeneer, Uppsala Univ. (Sweden); M. Münzenberg, Georg-August-Univ. Göttingen (Germany)

898417 Quantum tricks with femtosecond light pulses teach magnetic devices to think ultrafast (Invited Paper) [8984-42]

I. E. Perakis, Univ. of Crete (Greece) and Foundation for Research and Technology-Hellas (Greece); P. C. Lingos, Univ. of Crete (Greece); J. Wang, Ames Lab., lowa State Univ. (United States)

898418 Optical tailoring of electron and hole spin polarization in bulk germanium (Best Student Paper Award) [8984-43]

J. Lohrenz, T. Paschen, C. Hautmann, M. Betz, Technische Univ. Dortmund (Germany)

\section{SESSION 12 STRONG-FIELD PHENOMENA I}

8984 1A Extreme nonlinear optical processes with beams carrying orbital angular momentum (Invited Paper) [8984-45]

C. Kern, M. Zürch, P. Hansinger, Institute of Optics and Quantum Electronics, Abbe Ctr. of Photonics, Friedrich-Schiller-Univ. Jena (Germany); A. Dreischuh, Sofia Univ. (Bulgaria); Ch. Spielmann, Institute of Optics and Quantum Electronics, Abbe Ctr. of Photonics, Friedrich-Schiller-Univ. Jena (Germany) and Helmholtzinstitut Jena (Germany)

8984 1B Extending HHG spectroscopy to new molecular species [8984-46]

F. McGrath, P. Hawkins, E. Simpson, T. Siegel, Z. Diveki, D. Austin, A. Zaïr, Imperial College London (United Kingdom); M. Castillejo, Consejo Superior de Investigaciones Científicas (Spain); J. P. Marangos, Imperial College London (United Kingdom)

\section{SESSION 13 STRONG-FIELD PHENOMENA II}

$8984 \mathrm{lE} \quad$ Study of filamentation compression in the near infrared in the $1.6 \mu \mathrm{m}$ to $2 \mu \mathrm{m}$ region for HHG experiments (Best Student Paper Award) [8984-49]

S. Driever, Imperial College London (United Kingdom); J.-C. Delagnes, N. Fedorov, CEA, CELIA, CNRS, Univ. de Bordeaux (France); M. Arnold, K. Holzner, Imperial College London (United Kingdom); F. Burgy, D. Descamp, E. Cormier, CEA, CELIA, CNRS, Univ. de Bordeaux (France); D. Bigourd, A. Zaïr, Imperial College London (United Kingdom); E. Constant, CEA, CELIA, CNRS, Univ. de Bordeaux (France) 


\section{SESSION 14 NANOPLASMONICS II}

8984 IG Engineering plasmonic and dielectric directional nanoantennas [8984-52]

A. Hildebrandt, M. Reichelt, T. Meier, J. Förstner, Univ. Paderborn (Germany)

POSTER SESSION

$8984 \mathrm{lJ} \quad$ Ultrafast fluorescence polarization spectroscopy of near infrared dye in picosecond dynamic range: model and simulation [8984-55]

Y. Pu, Univ. of California, Irvine (United States)

Author Index 


\section{Conference Committee}

Symposium Chairs

David L. Andrews, University of East Anglia Norwich (United Kingdom)

Alexei L. Glebov, OptiGrate Corporation (United States)

Symposium Co-chairs

Jean Emmanuel Broquin, IMEP-LAHC (France)

Shibin Jiang, AdValue Photonics, Inc. (United States)

Program Track Chair

James G. Grote, Air Force Research Laboratory (United States)

Conference Chairs

Markus Betz, Technische Universität Dortmund (Germany)

Abdulhakem Y. Elezzabi, University of Alberta (Canada)

Jin-Joo Song, University of California, San Diego (United States)

Kong-Thon Tsen, Arizona State University (United States)

Conference Program Committee

Mischa Bonn, FOM Institute for Atomic and Molecular Physics

(Netherlands)

Alan D. Bristow, West Virginia University (United States)

Yujie J. Ding, Lehigh University (United States)

Kazuhiko Hirakawa, The University of Tokyo (Japan)

Rupert Huber, Universität Regensburg (Germany)

Robert A. Kaindl, Lawrence Berkeley National Laboratory (United States)

Dai-Sik Kim, Seoul National University (Korea, Republic of)

Torsten Meier, Universität Paderborn (Germany)

Walter Pfeiffer, Universität Bielefeld (Germany)

Mark I. Stockman, Georgia State University (United States)

Chi-Kuang Sun, National Taiwan University (Taiwan)

Fabrice Vallee, Université Claude Bernard Lyon 1 (France)

Klaas Wynne, University of Glasgow (United Kingdom) 
Session Chairs

1 Nonlinear Optical Phenomena

Markus Betz, Technische Universität Dortmund (Germany)

2 Active Plasmonics

Jacob B. Khurgin, Johns Hopkins University (United States)

$3 \mathrm{THz}$ Phenomena

Abdulhakem Y. Elezzabi, University of Alberta (Canada)

4 Nanoplasmonics I

Volker J. Sorger, The George Washington University (United States)

5 Ultrafast Dynamics in Carbon Nanomaterials

Markus Betz, Technische Universität Dortmund (Germany)

6 Coherent Optical Phenomena I

Ermin Malic, Technische Universitäł Berlin (Germany)

7 Coherent Optical Phenomena II

Christoph Lienau, Carl von Ossietzky Universität Oldenburg (Germany)

8 Nanophotonics

Xiaoqin Li, The University of Texas at Austin (United States)

9 Cavity-Related Phenomena

Walter Pfeiffer, Universität Bielefeld (Germany)

$10 \mathrm{THz}$ Detection and Imaging

Tobias Kampfrath, Fritz-Haber-Institut der Max-Planck-Gesellschaft (Germany)

11 Spins Dynamics and Ultrafast Magnetism

Yoan Léger, Institut National des Sciences Appliquées de Rennes (France)

12 Strong-Field Phenomena I

Peter Dombi, Max-Planck-Institut für Quantenoptik (Germany)

13 Strong-Field Phenomena II

Christian Spielmann, Abbe School of Photonics (Germany)

14 Nanoplasmonics II

Abdulhakem Y. Elezzabi, University of Alberta (Canada) 